\title{
Motion Blur and BP Algorithm Network Integrated for the Control and Diagnosis of Automobile Sensor Fault
}

\author{
Tuo Chaoyong \\ (Hunan Mechanic \& Electric Polytechnic, Changsha Hunan 410151) \\ 2011583056@qq.com
}

\begin{abstract}
With regard to the fault control and diagnosis objects of the automobile sensors, the problems include the large space dimension of the fault sample data, the poor timeliness of fault diagnosis, the rather poor ability to identify the diagnosis conclusion and the uncertainty etc. In this paper, it is presented to adopt the Dynamic Blur Self-learning theory combined with the BP Neural Network to diagnose the faults. First, the Dynamic Blur Self-learning method is used to find the fault nodes, and then the BP Neural Network is used to carry out quick classified diagnosis. The simulation research shows that this diagnosis method can not only keep the accuracy rate of sensor fault diagnosis and improve its speed, but also reduce the uncertainty of fault conclusion and improve the performance of the diagnosis in identification and decision making.
\end{abstract}

Keywords: Dynamic Blur, BP Neural Network, sensor fault

\section{Introduction}

Along with the improvement of people's living standards, the car has become an important part of people's lives. Literature[1] pointed out that the extensive use of computer and information technology into the car, where the sensor is gradually applied to automotive control, which can perceive, acquire, converse and process automotive information. The perceptive information needed will be converted into other information and get output. Automotive sensor is a key component of automotive electronic control, as well as a core part of automotive electronic technology.

Literature [2-3] pointed out that when encounters the car sensor failure, the car sensor will judge for car trouble through its own control systems and aids, but due to the current automotive sensor control frequently fails, therefore people need a convenient and efficient engines sensor failure control method. Literature [4] noted that the current sensor control is mainly distributed against the bottom of scale body: it accomplishes faulty signal output through the sensor output, but the easy off-center affects estimation accuracy. Literature [5] pointed out that the digital load cell can achieve uninterrupted work, and the fault signal can be obtained in a short time, but the drawback is expensive. Literature [6-7] pointed out that the use of neural networks to car failure reasoning, using priority and setting weights and other methods for fault diagnosis, but these methods need to be supported by the expert system, hence have a certain dependence.Literature [8 ] proposed a Bayesian network proposed fault diagnosis method based on multi- sensor data fusion: the experiment shows that the method improves the diagnostic accuracy and has good application value. Literature [9 ] proposed a sensor fault diagnosis expert system based on neural network fusion, which integrated BP neural network for fault detection; the findings indicate that the sensor diagnosis expert system has high accuracy and fast diagnosis characteristics. Literature [10 ] proposed a new method of sensor nodes fault diagnosis based on rough sets and ant colony optimization neural networks, and the simulation shows that the method has a high diagnostic accuracy and efficiency. 
Literature [11 ] proposed a method for automotive sensor fault simulation system. The simulation results were compared with the real vehicle test results, which show that using this method to establish the system meets the test requirements, and is easy to use with high reliability and high accuracy. Literature [12 ] proposed a sensor fault diagnosis method based on wavelet transform and neural network method, which can effectively distinguishes signal changes caused by signal sensor failure between normal fluctuations changes caused by the process itself ; in training the neural network system it requires only a sample of normal state, which overcomes the difficulties of the scarce sensor fault samples. Experimental results show that this method can effectively complete fault diagnosis and can determine the sensor fault type.

This paper has combined the motion blur function and BP neural network for effective and accurate classification of the car sensor control failure. The paper also conducts accurate diagnosis and recovery for automotive sensors fault, which can be effectively used to ensure the normal operation of automotive sensors.

\section{Car sensor Control Fault Model}

The output signal is mainly automotive sensor voltage signal, when the wiring between automotive sensors and $E C U$ (sensor and the engine control unit) have open circuit, the voltage signal will be beyond the normal range and thus causes a malfunction. Usually we set the car's normal range of the sensor output signal voltage is $\left(U_{\min }, U_{\max }\right)$, if the actual input signal voltage is greater than $U_{\max }$ or less than $U_{\text {min }}$, it is considered that the signal is not reliable, which means that the sensor is faulty. Only after the sensor signal lasts some time, a fault in ECU is judged. Assuming the number of sensors in the vehicle sensor network nodes is $J$, each node in the data acquisition sampling process samples for $n$ times, the data length of a single node is ${ }^{n}$. Data collected as a single node in the column of the matrix, the network data may be represented as

$\boldsymbol{X}_{2 D}=\left(\begin{array}{ccc}x_{11} & \cdots & x_{1 J} \\ \vdots & \ddots & \vdots \\ x_{n 1} & \cdots & x_{n J}\end{array}\right)$

For ease of presentation, data in each node will be converged, and network data can be written in vector form

$$
\begin{aligned}
\boldsymbol{X} & =\operatorname{vec}\left(\boldsymbol{X}_{2 D}\right) \\
& =\left(x_{11}, \ldots, x_{1 n}, x_{12}, \ldots, x_{n 2}, x_{1 J}, \ldots, x_{n J}\right)^{T}=(x(1), x(2), \ldots x(N))^{T}
\end{aligned}
$$

Wherein $\boldsymbol{X} \in R^{N}, \quad N=J \times n$.

Before compressing and sensing measurements of the data, we use discrete cosine transform (Discrete Cosine Transform, DCT) for converting the sparse voltage signal

$$
\boldsymbol{X}=\boldsymbol{\Psi} \boldsymbol{S}=\sum_{i=1}^{N} \psi_{i} s_{i}=\sum_{k=1}^{K} \psi_{k} s_{k}, s_{k} \neq 0
$$

Wherein $\boldsymbol{\Psi}$ is $N \times N$ dimensional DCT transform matrix; in $N$ dimensional sparse coefficient vector ${ }^{s_{k}}$ contains $K \quad(K \ll N)$ nonzero elements. Measurement data measured by the matrix is generated by the local node, denoted node ${ }^{j}(j \in 1,2, \ldots, J)$ 
generated the measurement matrix as $\boldsymbol{\Phi}_{j}$, the matrix of dimension is $m \times n(m<<n)$. The resulting measured value of each node is written as a vector form

$\boldsymbol{Y}=\left(y_{11}, \ldots, y_{1 m}, y_{12}, \ldots, y_{m 2}, y_{1 J}, \ldots, y_{m J}\right)^{T}$

$=(y(1), y(2), \ldots y(M))^{T}$

The measurement process of network data matrix can be expressed in vector form as follows:

$\boldsymbol{Y}=\boldsymbol{\Theta X}=\boldsymbol{\Phi} \Psi \boldsymbol{s}$

wherein $\boldsymbol{Y} \in R^{M}, M=J \times m$, Measurement matrix

$$
\boldsymbol{\Phi}=\left(\begin{array}{cccc}
\Phi_{1} & 0 & \cdots & 0 \\
0 & \Phi_{2} & \cdots & 0 \\
\vdots & \vdots & \ddots & \vdots \\
0 & 0 & \cdots & \Phi_{J}
\end{array}\right)_{\in R^{M \times N}}
$$

In order to find the vehicle sensor control nodes data timely and accurately, we need to use known measured value $Y$ and under restoring matrix $\boldsymbol{\Theta}$ conditions to reconstruct sparse coefficients matrix vector. As the vehicle sensor data sparse coefficient vector control node sparse degree changes with node mobility, this paper will propose a new method to solve the fault diagnosis for this problem.

\section{Automotive Sensor Diagnostic Control based on Dynamic Fuzzy and BP Neural Network Algorithm}

\subsection{Energy Self-Learning based on Dynamic Fuzzy Function}

Automotive sensor network failure has some randomness, and it is a typical nonlinear structure; whereas dynamic fussy function may well find death nodes in sensor fault. Dynamic fuzzy basis function is constructed as follows:

$$
F_{n}(x)=\frac{\prod_{i=1}^{n} T_{i}(x)}{\sum_{i=1, n=n}^{n} \prod_{i=1}^{n} T_{i}(x)}=\frac{r u q\left[\sum_{i=1, j=1}^{n}\left(\frac{x_{i}-\overline{x_{j}}}{\sigma_{i j}}\right)^{2}\right]}{\sum_{i=1, j=1, \ldots n} r u q\left[-\sum_{i=1, j=1}^{n}\left(\frac{x_{i}-\overline{x_{j}}}{\sigma_{i j}}\right)^{2}\right]}
$$

Through setting car sensor nodes energy (set as $w_{i}$ ), we enter it into the formula (5) to give the corresponding node energy improvements as follows:

$$
Y=\sum_{i=1}^{k} F_{i}(x) \square w_{i}
$$

In the formula (6), in which $k$ represents the number of sensor nodes. By constructing dynamic functions in fuzzy function as follows: 


$$
\begin{aligned}
& \text { if } x_{i} \in T(x) \text { then } \\
& Y=\sum_{i=1}^{N} \frac{u_{i}}{y_{i}}
\end{aligned}
$$

In equation (7), $x_{i}$ as fuzzy variable, $T(x)$ means REFERENCES fuzzy variables. Wherein $u_{i}$ is set as real numbers between 0 and $1, \frac{u_{i}}{y_{i}}$ Indicates the possibility of $y_{i}$ is $u_{i}$; meaning : when $x_{i}$ reaches $T(x)$, the likelihood of $y_{i}$ is $u_{i}$. Assuming $T(x)=\left\{u_{1} \cdots u_{n}\right\}$ contains $n$ specifications, we set $T$ represented by mamdani implication, by $T_{1}^{\prime} \cdots T_{n}^{\prime}$ using mamdani to infer $T^{\prime}=\left(\alpha_{1} u_{1} \cdots \cdots \alpha_{n} u_{n}\right)$. Using Equation (8) to conduct self-learning $T^{\prime}$ and we obtain $T^{\prime \prime}$, whose precision is much larger than $T^{\prime}$.

$$
T^{\prime \prime}=\frac{\sum_{i=1}^{N} Y_{i} \alpha_{i} u_{i}}{\sum_{i=1}^{N} \alpha_{i} u_{i}}
$$

We combine three Equation (6), (7) and (8) to obtain a self-learning ability to dynamically control functions for automotive sensor nodes, thereby enabling rapid judgment on automotive sensor nodes energy loss.

\subsection{BP Neural Network}

BP neural network is a one-way transmission network, usually consists by the input layer, hidden layer and output layer. It spread signal in forward and reverse transmission. In reverse transmission, weights are adjusted by Delta learning rule. In forward transmission, we sequentially according to formula (9) in the input and output of each layer until the output layer. When the output layer is not expected, it conducts reverse transmission according to the error adjustment weights and thresholds between expectations and actual output. See formula (10) for adjustment formula weights.

$$
\begin{aligned}
W_{i} & =\sum_{j} w_{i j} x_{j}+\theta_{i} \\
y_{i} & =f\left(W_{i}\right)
\end{aligned}
$$

In the formula (1),$W_{i}$ is the activation value of the $i$-th layer node, $\theta_{i}$ is the threshold value, $x_{j}$ is the input signal, $w_{i j}$ is the connection weights coefficient of the first node and the second node, and $y_{i}$ is the output value of the node $i$.

$$
w_{i j}(t+1)=w_{i j}(t)+\frac{\partial E}{\partial w_{i j}}
$$

In formula (10), $\frac{\partial E}{\partial w_{i j}}$ is the error between the desired output of neural network and the actual output.

\subsection{Description of the Algorithm}

In this paper, it uses the sparsity in a transform domain to achieve control of 
automotive sensors detect reconstruction algorithm. Most of the current reconstruction algorithm combine sparse vector sparse pre-specified level of prior information to support the potential size of the set, whereas the algorithm in this paper based on this method, first to determine the set of automotive sensor failures through self-study in support of the dynamic function, and then through BP neural network algorithm of fault sensor for rapid classification, thus shortening the detection time to improve the detection efficiency.

Specific steps are as follows:

Initialization: residuals $\boldsymbol{r}_{0}=\boldsymbol{Y}$, iterations $t=1$, potential support set iteration step size $\neq 0$, the support set potential $L=$ size , segmentation number stage $=1$, the index set $\Lambda_{0}=\phi \Omega_{0}=\phi$.

(1) calculate the correlation coefficient between recovery matrix for each column and residuals, and select $2 L$ correlation coefficient that have the largest magnitude

$$
u_{t}=\left\{u_{i}\left|u_{i}=\right|\left\langle r, \Theta_{i}\right\rangle \mid\right\} \quad i=1,2, \ldots N, \Omega_{t_{0}}=\left(u_{t}, 2 L\right)
$$

(2) update the set of index values, and introduce into the formula (5)

$$
\begin{array}{ll}
\Lambda_{t-1}=\Lambda_{t-1} \square F_{n}(x) & \\
\Lambda_{t_{0}}=\Lambda_{t-1} \cup \Omega_{t} & \Lambda_{t}=\left(\Lambda_{t_{0}}, L\right)
\end{array}
$$

(3) estimated sparse coefficient vector

$$
\hat{\boldsymbol{s}}=\arg \min _{i \in R^{\wedge}}\left\|Y-\Theta_{A_{t}} s\right\|_{2}
$$

(4) Update the residuals: $\boldsymbol{r}_{\boldsymbol{t}}=\boldsymbol{y}-\boldsymbol{\Theta}_{\boldsymbol{A}_{t}} \hat{\boldsymbol{s}}$

(5) introduce into the formula (9) and formula (10), determines the algorithm termination condition: $\left\|\hat{\mathbf{s}}_{\mathbf{t}-1} \square_{y_{i}}-\hat{\mathbf{s}}_{\mathbf{t}}\right\|_{2}<\varepsilon$. If satisfied, the algorithm stops, output $\hat{\mathbf{x}}=\boldsymbol{\Psi} \hat{\mathbf{s}}_{w_{i j}}$; if not satisfied, go to Step

(6) continue this subparagraph iteration: $t=t+1$, to step (1).

Final solution: $\hat{\mathbf{x}}=\boldsymbol{\Psi} \hat{\mathbf{s}} \square_{w}$

\section{Test Simulation and Analysis}

In the Matlab environment, we first use 200 data samples in the original automotive sensor fault sample to design faults samples, each divided into 50 groups of data, the top 30 group for training, and the remaining 20 groups for testing. Then to sort fault samples through dynamic fussy function, while designing a BP neural network classifier, in order to verify the effect of dynamic fussy function of self-learning. Two BP neural network classifiers diagnostic results comparison is shown in Table 1. Three sets of data selected under impact sensor failure as shown in Table 2, after the dynamic function selflearning process we get Table 3, and BP neural network classifier actual output data is shown in Table 4.

Table 1. Comparison of Diagnostic Results

\begin{tabular}{ccccc}
\hline $\begin{array}{c}\text { The } \\
\text { input } \\
\text { layer }\end{array}$ & $\begin{array}{c}\text { hidden } \\
\text { layer }\end{array}$ & $\begin{array}{c}\text { output } \\
\text { layer }\end{array}$ & $\begin{array}{c}\text { training } \\
\text { times }\end{array}$ & $\begin{array}{c}\text { recogni } \\
\text { tion } \\
\text { rate }\end{array}$ \\
\hline 20 & 20 & 10 & 200 & $42 / 50$ \\
\hline 9 & 20 & 5 & 100 & $40 / 50$ \\
\hline
\end{tabular}


Table 2. Select three Fault Data Under Impact

\begin{tabular}{ccc}
\hline Group1 & Group2 & Group3 \\
\hline 0.25 & 0.28 & 0.47 \\
0.36 & 0.34 & 0.25 \\
0.38 & 0.42 & 0.48 \\
0.40 & 0.56 & 0.78 \\
0.93 & 0.88 & 0.91 \\
0.86 & 0.51 & 0.76 \\
0.68 & 0.47 & 0.56 \\
0.40 & 0.38 & 0.45 \\
0.49 & 0.35 & 0.32 \\
0.39 & 0.32 & 0.49 \\
0.45 & 0.35 & 0.37 \\
0.37 & 0.41 & 0.45 \\
\hline
\end{tabular}

Table 3. The Failure Data after Dynamic Fuzzy Functions Processing

\begin{tabular}{ccc}
\hline Group1 & Group2 & Group3 \\
\hline 1.5218 & 1.3568 & 1.6211 \\
-0.2118 & 0.0758 & 0.1848 \\
0.2108 & 0.2555 & -0.6081 \\
0.1725 & 0.0472 & -0.0046 \\
-0.0686 & 0.0156 & 0.1913 \\
-0.2248 & -0.3423 & -0.3551 \\
-0.2789 & 0.0041 & -0.2991 \\
0.0364 & -0.0629 & -0.0605 \\
\hline
\end{tabular}

Table 4. Neural Network Classifier Actual Output Data

\begin{tabular}{cccccc}
\hline Gr & & & & & \\
ou & Impact & stuck & offset & circle & normal \\
$\mathrm{p}$ & & & & & \\
\hline 1 & 0.5233 & 0.1875 & 0.0458 & 0.0704 & 0.3892 \\
2 & 0.4815 & 0.0751 & 0.0885 & 0.0483 & 0.4524 \\
3 & 0.6625 & 0.0287 & 0.0913 & 0.0472 & 0.3482 \\
\hline
\end{tabular}

From Table 1-4 we find after using using dynamic fussy function to process automotive sensor fault data sample, the input layer of the neural network was reduced from 20 to 8 , training times significantly reduced to 100 times; apparently CPU consuming time has reduced. And fault identification rate remained unchanged. It can be seen: the use of dynamic fuzzy functions ensures the recognition rate, but also simplifies the structure of BP neural network, improves the speed of diagnosis, which is method that could increase real-time-ness of BP neural network fault samples classification.

To further illustrate the superiority of the proposed method, the algorithm here mentioned and other algorithms are compared in terms of the detection error rate and detection failure rate. The results are shown in Figure 1-3. 


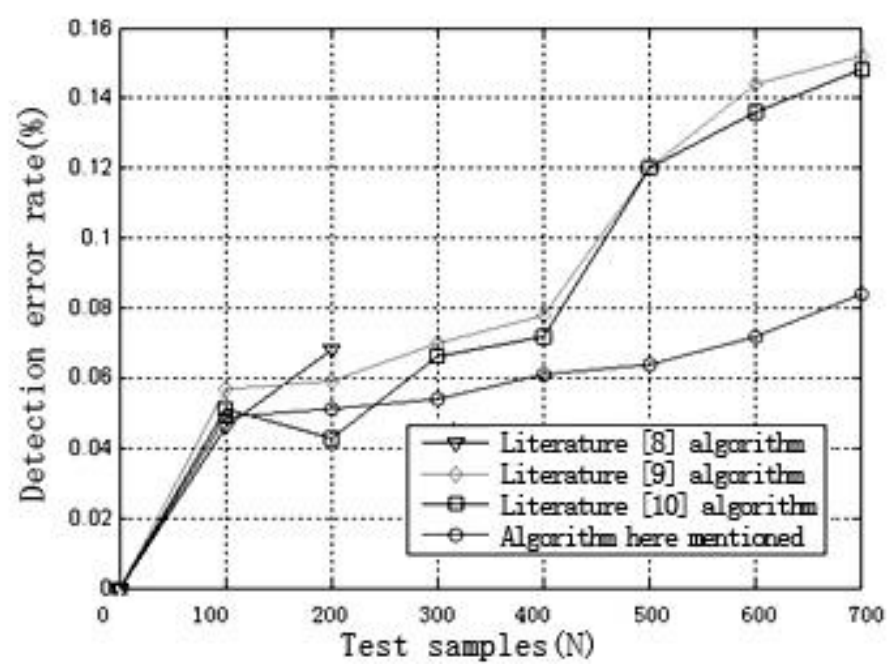

Figure 1. Detection Error Contrast in Four Algorithm

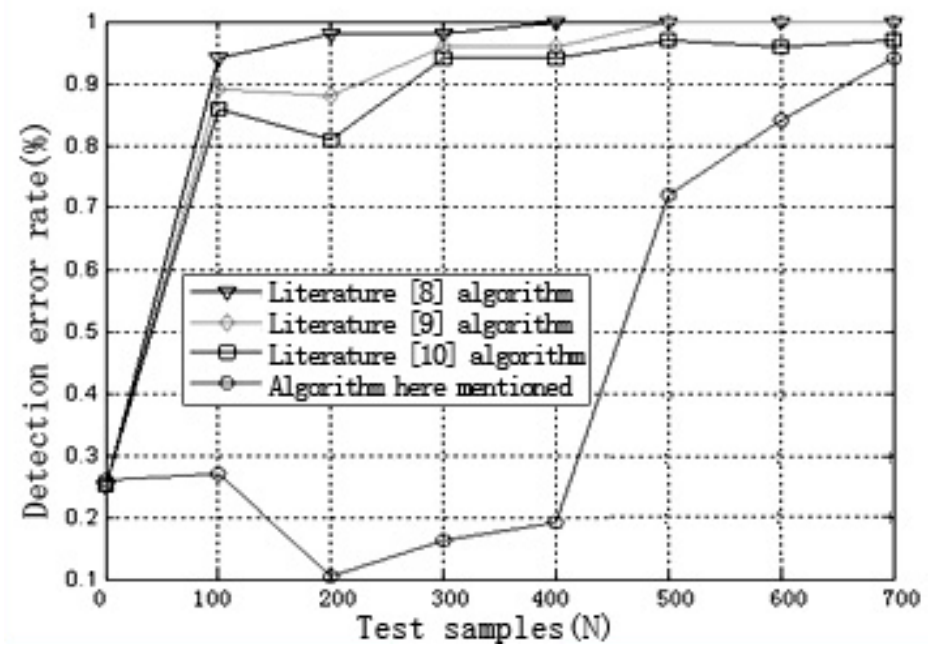

Figure 2. Detection Failure Rate Comparison in Four Algorithm

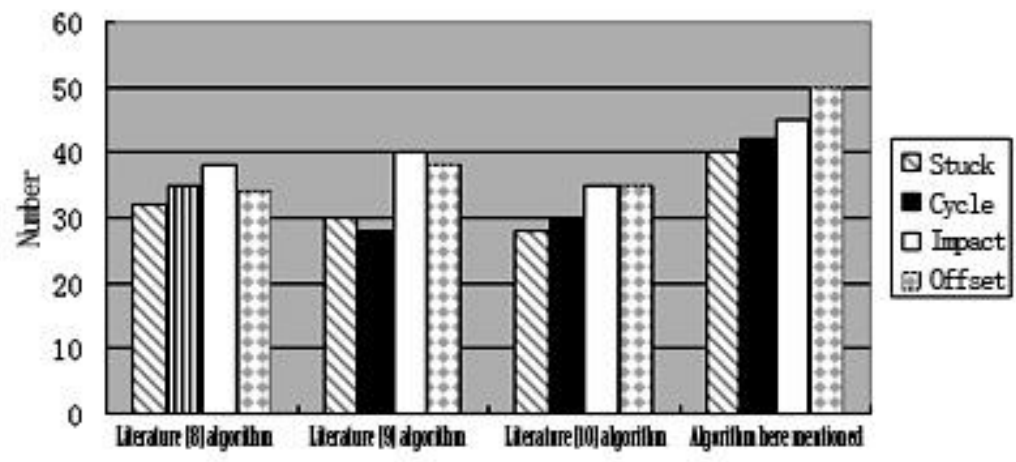

Figure 3. Comparison of Automotive Sensors Fault Detection Commonly used in Four Algorithm

As can be seen from the above three figures, the algorithm of this paper in terms of the measurement error is far greater than other three algorithms, effectively improves the accuracy of detection. In addition, the algorithm of this paper has a lower detection failure 
rate than the other three algorithms. In four faults detection, algorithm used in this paper is superior to the other three, which shows that the algorithm has certain advantages.

\section{Conclusion}

For automotive sensor fault detection control, we propose a dynamic fuzzy function and BP neural network-based method for rapid classification of faults: first for the input data of fault sample we use dynamic fuzzy function for data collection. And then use output of the neural network data for data classification. The simulation results show that: the algorithm of this paper has ensured the accuracy of the fault detection, while simplified the structure of neural network, improved the speed of fault diagnosis. A combination of dynamic fuzzy function and BP neural network used in automotive sensors control fault diagnosis is feasible.

\section{References}

[1] Zhao Jiong, etc. To develop electric vehicles autopilot system based on sensor fusion technology [J]. Manufacturing automation, 2013.35 (5) : $43-46$

[2] Qiu Xiao hong, etc. Fault diagnosis technology based on Modelical extended modeling [J]. Technologies Bulletin, 2011,27 (5) :36-39

[3] Wang Qian, etc. Summary of K-means clustering algorithm [J]. Electronic design engineering, 2012,20 (7) : $40-43$

[4] BLISS D,STICKEL C,BENTZ J W.Load cell diagnostics and failure prediction weighing apparatus and process[P].Uunited State Patent:728638,2000

[5] PENG X F.Application of digital load cells[J].Industrial Measurement,2008,(01):62-63

[6] JIANMING L,Yonggon L,SCOTT D S,et al.Selforganizing radial basis function network for real-time approximation of continuous-time dynamical systems[J].IEEE Trans.Neural Networks, 2008,19(3):460-474

[7] Wang Luo, etc. Regression vector support machine in motor fault diagnosis research [J]. Computer Measurement \& Control, 2007,15 (2) :1691-1694

[8] Liu Xiliang, etc. Research and Application of Bayesian network fault diagnosis based on multi-sensor information fusion [J], Mechanical Science and Technology, 2013,32 (1) :91-95

[9] Wei, Hongjuan Sensor fault diagnosis expert system based fusion neural network [J]. Computer Measurement \& Control, 2013,21 (2) :358-361

[10] Fu, Zhongjie. Rough sets and ant colony neural network applied in diagnosis sensor node failure [J] . Computer Measurement \& Control, 2012,20 (10):2628-2631

[11] Zhou Bing, etc. Engine sensor fault hardware in the loop simulation platform [J]. Chinese Mechanical Engineering, 2013,24 (5) :1181-1184

[12] Zhao Jinsong A sensor fault diagnosis method based on wavelet transform and neural network [J] Journal of Tsinghua University (Natural Science) .2013,53 (2) :205-209

\section{Authors}

Tuo Chaoyong (1962.5-), male, associate professor, research direction: automatic control, auto fault diagnosis.

为方便组委会联系, 请提供 2 位作者信息。

\begin{tabular}{|c|c|c|c|}
\hline 论文题目 & \multicolumn{3}{|c|}{ 基于动态模糊与 $\mathrm{BP}$ 神经网络的汽车传感器故障控制诊断 } \\
\hline 所属主题 & \multicolumn{3}{|c|}{ 其他主题 } \\
\hline \multicolumn{4}{|c|}{ 第一作者 } \\
\hline 姓名 & 席朝永 & 职称/学位 & 副教授 \\
\hline 单位 & 湖南机电职业技术学院 & 邮编 & 410150 \\
\hline
\end{tabular}




\begin{tabular}{|c|c|c|c|}
\hline 地址 & \multicolumn{3}{|c|}{ 长沙市开福区万家丽北路一段 359 号 } \\
\hline 电话 & & 手机 & 13735242370 \\
\hline Email & \multicolumn{3}{|c|}{ 2011583056@qq.com } \\
\hline \multicolumn{4}{|c|}{ 第二作者 } \\
\hline 姓名 & & 职称/学位 & \\
\hline 单位 & & 邮编 & \\
\hline \multicolumn{4}{|l|}{ 地址 } \\
\hline 电话 & & 手机 & \\
\hline Email & & & \\
\hline
\end{tabular}


International Journal of Control and Automation Vol.7, No.8 (2014) 